\title{
Family tree and population: Distinction and similarity of the analysis on example of hemophilia
}

\author{
Andrey N. Volobuev, Peter I. Romanchuk, Vladimir K. Malishev
}

Samara State Medical University, Samara, Russia; volobuev47@yandex.ru

Received 24 June 2013; revised 24 July 2013; accepted 1 August 2013

Copyright (C) 2013 Andrey N. Volobuev et al. This is an open access article distributed under the Creative Commons Attribution License, which permits unrestricted use, distribution, and reproduction in any medium, provided the original work is properly cited.

\begin{abstract}
On the basis of Hardy-Weinberg law the distinction in the philosophy of the description of a family tree and a population is considered. With the help of introduction of the equivalent constant mutagen factor both in a family tree and in a population, the analysis of hemophilia is led. Influence of selection at presence of hemophilia is considered. On the basis of idea of Danforth and Haldane about the balance mutagenesis and selection at presence of hemophilia, the interrelation of selection parameter and the equivalent constant mutagen factor is found. It is shown, that dynamics of development of hemophilia in a family tree and in population are similar.
\end{abstract}

Keywords: Alleles; Equivalent Constant Mutagen Factor; Family Tree; Population; Hemophilia; Balance of Selection and Mutagenesis

\section{INTRODUCTION}

Mathematical genetics is a completely special section of biophysics. It enables to understand those or other phenomena in human community, to explain and predict development of this community as from the point of view of health of people, and social processes in community. It allows us to understand the purposes and problems of human community for improvement of the life.

The mathematical genetics is based on Hardy-Weinberg law which was found by English mathematician Hardy and German doctor Weinberg in 1908.

This law in the elementary kind of two alleles of a gene determines that relative frequencies of genotypes in generations correspond to terms of binomial expansion $(p+q)^{2}$, so $p+q=1$ where $p$ and $q$ there are alleles frequencies in a population or that is more correct in a family tree. Relative frequencies of genotypes remain constant from generation to generation in case of an ideal population (number of individuals is very large, there is pan-mixing, there is no selection, mutation, migration of individuals, etc.) [1].

The population will consist of set of family trees which periodically contact among themselves.

The exponent 2 specifies that two different-sex species are necessary for reception of posterity. And the persons are the man and the woman.

Hardy-Weinberg law in genetics has the same fundamental role as, for example, 2-nd Newton law in mechanics. But in the relation to world around these two laws of genetics and mechanics there is an important distinction. The exponent 2 at the Hardy-Weinberg law reflects concrete conditions of life occurrence in the Earth. It is possible to assume that on other manned planets it exponent can have other value. If three different-sex species, for example, are necessary for reception of posterity at the Hardy-Weinberg law there should be an exponent 3 . The second Newton law undoubtedly has the same kind everywhere in Universe.

Extremely important topic of mathematical genetics is that there are mutations. The mutations explain genetically dependent hereditary diseases. Mutations allow to explain process of evolution of organisms. Mutations underlie animals and plants breeding.

Mutations are spontaneous and induced [2].

During induced mutations there is an interaction of the mutagen factor and a species with an exposed mutation.

Process of a mutation has stochastic character. The species under action of the mutagen factor with some probability can be subjected to mutations, and can not be subjected.

During the mutation the mutagen factor has the important role. It is possible to classify mutagen factors into two groups: determined and stochastic.

The determined mutagen factors can be constant or functionally time-dependent.

There is pertinently to present the following mechanical analogy. 
In a room there is an air ball-analogue of the mutagen factor. Molecules of air in a room play a role of species. The model mutation is an impact of a molecule with a ball.

The probability of a mutation depends on a state of a molecule, in this case distances from a ball. With a ball it can cooperate, and the distant molecule and a near molecule can depart from a ball during thermal movement not having heat around it.

But the probability of a mutation depends on behavior of a ball.

The ball can not change the volume - the constant mutagen factor.

It can increase at blow-up and reduce at blow-away. The probability of mutations is changing. For example, at full blow-away a ball probability of mutations become equal to zero. The ball can periodically or aperiodically change the volume. All these processes with a ball are model of the determined mutagen factors [3].

The ball also can change the volume completely stochastic and it is the model of stochastic mutagen factor [4].

\section{ACTION OF THE CONSTANT MUTAGEN FACTOR ON A COUPLED WITH THE X-CHROMOSOME GENOME}

The intensive use of the mobile communication, insufficient protection against electromagnetic radiation of computers on a workplace result to that there is a constant mutagen electromagnetic background which is a source of the changes touching a genofund of a human population. Therefore is of interest to consider existence and development genome in conditions of influence of some constant mutagen factor.

At action of the constant mutagen factor there is probably occurrence of the selection resulting in change of genic frequencies in one direction [2].

Let's consider action of the constant mutagen factor on two-alleles genome coupled with the $X$-chromosome. For this purpose we shall assume, that alleles $A$ and $a$ are coupled with the $X$-chromosome. The frequency of dominant alleles $A$ we shall designate at men $p_{m}$ and at women $p_{f}$. For recessive alleles $a$ it is accordingly $q_{m}$ and $q_{f}$.

At crossing in the first generation there is a ratio of genotypes at women, according to product $\left(p_{f}+q_{f}\right)\left(p_{m}+\right.$ $\left.q_{m}\right)$. Thus:

$$
(A A) p_{f} p_{m}:(A a)\left(p_{m} q_{f}+p_{f} q_{m}\right):(a a) q_{m} q_{f} .
$$

Men have gemizygote on genes in the $X$-chromosome the frequency ratio determined by that the $X$-chromosome of the woman at crossing passes to the man's de- scendant:

$$
\text { (A) } p_{f}:(a) q_{f}
$$

Distribution (1) can be used also for the description of blood system ABO. In spite of the fact that to this system corresponds three-alleles ensemble of the genes the two alleles $A$ and $B$ are dominant and their general frequency can be designated at men $p_{m}$ and at women $p_{f}$. Alleles $\mathrm{O}$ has in this case frequency at men $q_{m}$ at women $q_{f}$. The ratio (1) for blood system $\mathrm{ABO}$ is not frequency distribution of genotypes of blood but the genotype frequency $a a$ (or a genotype OO), and also phenotype frequency corresponding to a blood group I it the ratio reflects truly. For example, frequency of alleles $\mathrm{O}$ at men and women in Berlin is equal $q_{f}=q_{m}=0,6057$, [2].

The basic demonstration of existence $X$-coupled recessive inheritance for a blood system ABO consists that the destruction at disease of blood, for example, hemophilia are men and daughters phenotypic are healthy.

For the first time the mathematical genetics laws has applied Haldane to a problem of hemophilia on basis of Danforth idea about an equilibration of frequency of mutations and selection. Occurrence of hemophilia there is usually concern to spontaneous mutations. However, formally meaning balance of mutations and selection, and also a constancy of occurrence of a family tree mutation (otherwise illness quickly would disappear) it is possible to calculate a task of a hemophilia assuming action of some equivalent constant mutagen factor. Action of selection will be appreciated further.

For a finding of recessive alleles $a$ frequency in a new generation it is necessary to add frequency of homozygote $a a$ and half of frequency of heterozygote $A a$ from a ratio (1). It is similarly necessary to act and for alleles $A$. Using this way of calculation of allele frequencies we find that change of alleles $a$ frequency in generations of women equally:

$$
q_{f n}=\frac{1}{2}\left[q_{f(n-1)}+q_{m(n-1)}\right] .
$$

Let's assume:

$$
\frac{1}{b} q_{m(n-1)}=q_{f(n-2)},
$$

where $b=\frac{q_{m(n-1)}}{q_{f(n-2)}}$ it is some constant factor which we shall name the constant mutagen factor. This factor specifies that alleles frequency $a$ at the woman of the previous generation not in accuracy is equal to alleles frequency at the man of the following generation. Such situation develops if alleles frequency $a$ in a female part of a population during to live before the reproductive period is reduced due to the arisen mutations resulting in 
impossibility to posterity. [2].

According to (4) Eq.3 becomes:

$$
q_{f n}=\frac{1}{2}\left[q_{f(n-1)}+b q_{f(n-2)}\right] \text {. }
$$

For the solution of the Eq.5 we shall search as $q_{f n}=a^{n}$ where $a$ there is constant. Substituting this formula in (5), we shall find:

$$
a^{n}=\frac{1}{2}\left[a^{n-1}+b a^{n-2}\right] .
$$

Having divided the both parts of the equation on $a^{n-2}$, we shall receive some characteristic equation:

$$
a^{2}-\frac{1}{2} a-\frac{b}{2}=0 \text {, }
$$

which solutions are two roots $a_{1}=\frac{1}{4}(1+\mu)$ and $a_{2}=\frac{1}{4}(1-\mu)$ where $\mu=\sqrt{1+8 b}$ we shall name the reduced constant mutagen factor. At $b=1$ or $\mu=3$ the expression (5) describes genome equilibrium condition at absence mutagenesis.

Hence the general solution of the Eq.5 looks like:

$$
q_{f n}=C_{1}\left(\frac{1}{4}(1+\mu)\right)^{n}+C_{2}\left(\frac{1}{4}(1-\mu)\right)^{n},
$$

where $C_{1}$ and $C_{2}$ there are constants. The solution (8) with account of initial conditions describes change of alleles $a$ frequency at women in generations with account of the mutagen factor action.

Constants $C_{1}$ and $C_{2}$ we shall find proceeding from initial conditions: at $n=0, q_{f n}=q_{f 0}$ and at $n=1$ according to (3) $q_{f n}=q_{f 1}=\frac{q_{m 0}+q_{f 0}}{2}$.

We believe that in zero generation of men and women action of the mutagen factor is absent. Thus:

$$
\begin{gathered}
C_{1}=\frac{2 q_{m 0}+(1+\mu) q_{f 0}}{2 \mu}, \\
C_{2}=-\frac{2 q_{m 0}+(1-\mu) q_{f 0}}{2 \mu} .
\end{gathered}
$$

Substituting constants $C_{1}$ and $C_{2}$ in the formula (8), we receive the solution of the Eq.5 as:

$$
\begin{aligned}
q_{f n}=\frac{1}{2 \mu 4^{n}} & \left(2 q_{m 0}\left((1+\mu)^{n}-(1-\mu)^{n}\right)+\right. \\
& \left.+q_{f 0}\left((1+\mu)^{n+1}-(1-\mu)^{n+1}\right)\right),
\end{aligned}
$$

where for the further transformations it is convenient to use the function of integer argument:

$$
F(n)=\frac{(1+\mu)^{n}-(1-\mu)^{n}}{4^{n}} .
$$

In this case, the formula (9) will be transformed to a kind:

$$
q_{f n}=\frac{1}{\mu}\left(q_{m 0} F(n)+2 q_{f 0} F(n+1)\right) .
$$

If number of generations there is $n \rightarrow \infty$ then at $\frac{1 \pm \mu}{4}<1$ a limit $\lim \left(\frac{1 \pm \mu}{4}\right)^{n} \rightarrow 0$ if $n$ is integer. In this case frequency $q_{f n} \rightarrow 0$. On Figure 1, the dependence plotted under the formula (11) of alleles $a$ frequency at women from number of generation $n$ at various values of the mutagen factor $b$ is shown. Initial alleles $a$ frequency at women was accepted $q_{f 0}=0.7$ at men $q_{m 0}=0.3$. Curve ABO is plotted for frequency of blood alleles $\mathrm{O}$ at women and initial frequencies of it alleles $q_{f 0}=q_{m 0}=0.6057$. The equivalent constant mutagen factor for curve ABO was accepted equal $b=0.8$.

From Figure 1, it is visible that at absence of the mutagen factor influence $b=1$ the alleles $a$ frequency at women gradually drawing near to equilibrium frequency $q_{f \infty}=\frac{q_{m 0}+2 q_{f 0}}{3}=0.57$ that coincides with [5]. At occurrence of the mutagen factor there is a decrease in alleles $a$ frequency and if all over again are observed jumps of frequency then there is a smooth decrease.

Let's find change of alleles $a$ frequency in generation of women:

$$
\begin{aligned}
& \Delta q_{f(n-1)}=q_{f n}-q_{f(n-1)} \\
& =\frac{1}{\mu}\left(\left(q_{m 0}-2 q_{f 0}\right) F(n)+2 q_{f 0} F(n+1)-q_{m 0} F(n-1)\right)
\end{aligned}
$$

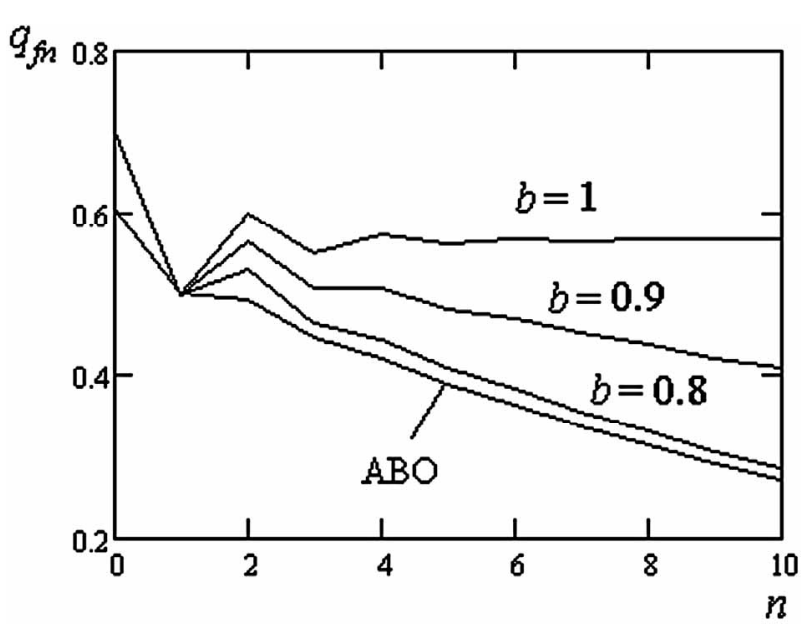

Figure 1. Dependence of alleles $a$ (or $\mathrm{O}$ ) frequency at women from number of generation $n$ at various sizes of the mutagen factor $b$. 
On Figure 2, the calculation of change of alleles $a$ frequency used under the formula (12) is shown depending on generation of women $n$ at various values of the mutagen factor $b$.

Apparently from Figure 2, the approach to equilibrium state of genome $\Delta q_{f(n-1)}=0$ is observed only at absence of the mutagen factor influence, i.e. at $b=1$. Otherwise there is a constant decrease in alleles $a$ frequency in generations. And approximately to the seventh generation the constancy of such decrease is established: in 10-th generation at $b=0$. size $\Delta q_{f(n-1)}=-0.014$, at $b=0.8$ size $\Delta q_{f(n-1)}=-0.021$.

On Figure 3, change of alleles blood $\mathrm{O}$ frequency is shown in generations of women at the equivalent constant mutagen factor $b=0.8$.

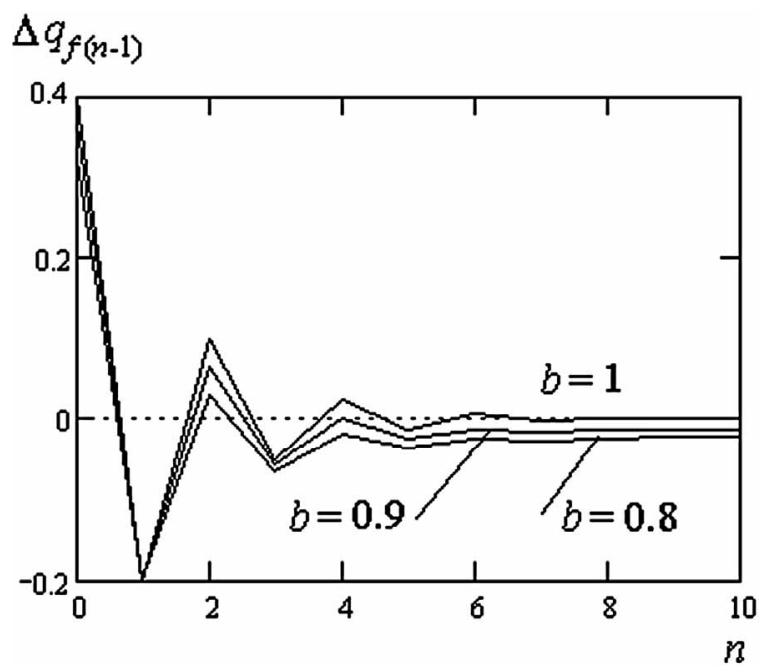

Figure 2. Change $\Delta q_{f(n-1)}$ of alleles $a$ frequency depending on number of generation of women at various values of the mutagen factor $b$.

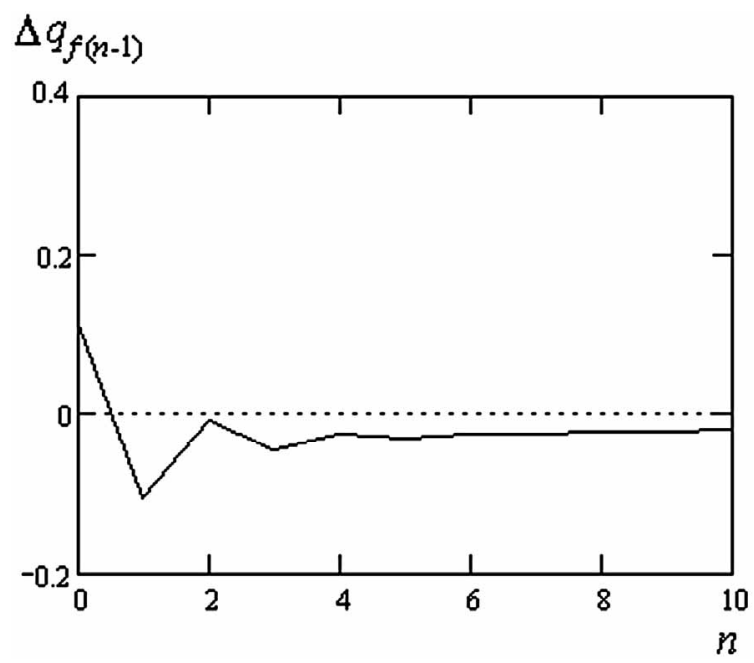

Figure 3. Change $\Delta q_{f(n-1)}$ of alleles blood $\mathrm{O}$ frequency in generations of women at the equivalent constant mutagen factor $b=0.8$.
The mutagen factor acting on alleles $\mathrm{O}$ can result, for example, in hemophilia. In 10-th generation, size $\Delta q_{f(n-1)}$ $=-0.02$. It specifies, that there is a gradual reduction of healthy alleles $\mathrm{O}$ frequency at women and during too time increase at them destructive alleles $\mathrm{O}$ frequency. According to the formula (11), see Figure 1, at the second generation healthy alleles $\mathrm{O}$ frequency at women to become equal $q_{f 2}=0.492$.

Hence, frequency destructive alleles has increased at them by size $0.6057-0.492=0.113$. Also increase frequency destructive alleles $\mathrm{O}$ at men of the third generation since it is transferred the man. At men destructive alleles $\mathrm{O}$ phenotypic it can be shown as hemophilia.

On Figure 4 according to [2], the family tree of the European royal houses in which men frequently were ill hemophilia is shown. Distribution of the $X$-chromosome in a family tree is shown also. The circle leads round the $X$-chromosome having destructive alleles $\mathrm{O}$ which causes hemophilia. Black squares there are designate men which are sick of hemophilia. From this family tree the gradual increase in number of men which are sick of hemophilia is well visible. The shown family tree not full since on it is possible to conclude that in the third generation all men are sick of hemophilia. Actually it is far from being so. And now in the given royal dynasty the share of sick hemophilia men are not too great. According to the submitted family tree in the first generation from four men one is sick of hemophilia, and in the second generation from seven men three are sick of hemophilia. Rough calculation of increase in frequency sick of hemophilia in a considered dynasty in the first and second generation at men has size $\frac{3}{7}-\frac{1}{4}=0.178$.

Corresponding reduction of a share of genotypic healthy women in the zero and first generation, counted up under the formula (12) is equal 0.106, see Figure 3. If should be more full family tree results apparently should be more close.

\section{CONNECTION BETWEEN STANDARD PARAMETER OF SELECTION AND THE CONSTANT MUTAGEN FACTOR}

In a family tree where the hemophilia is observed, there is selection resulting in decrease of genic frequencies in particular of alleles $\mathrm{O}$ to one direction.

Action of selection there is intensive enough. For example, the life period of the men which are sick of hemophilia makes $1 / 3$ from life period of healthy people [2]. Ability to leave offspring at men in comparison with healthy men is reduced. Therefore, not all men of a family tree take part in reception of offspring and destructive alleles $\mathrm{O}$ eliminate from a family tree. However is possible the balance of mutagenesis and selection resulting to 


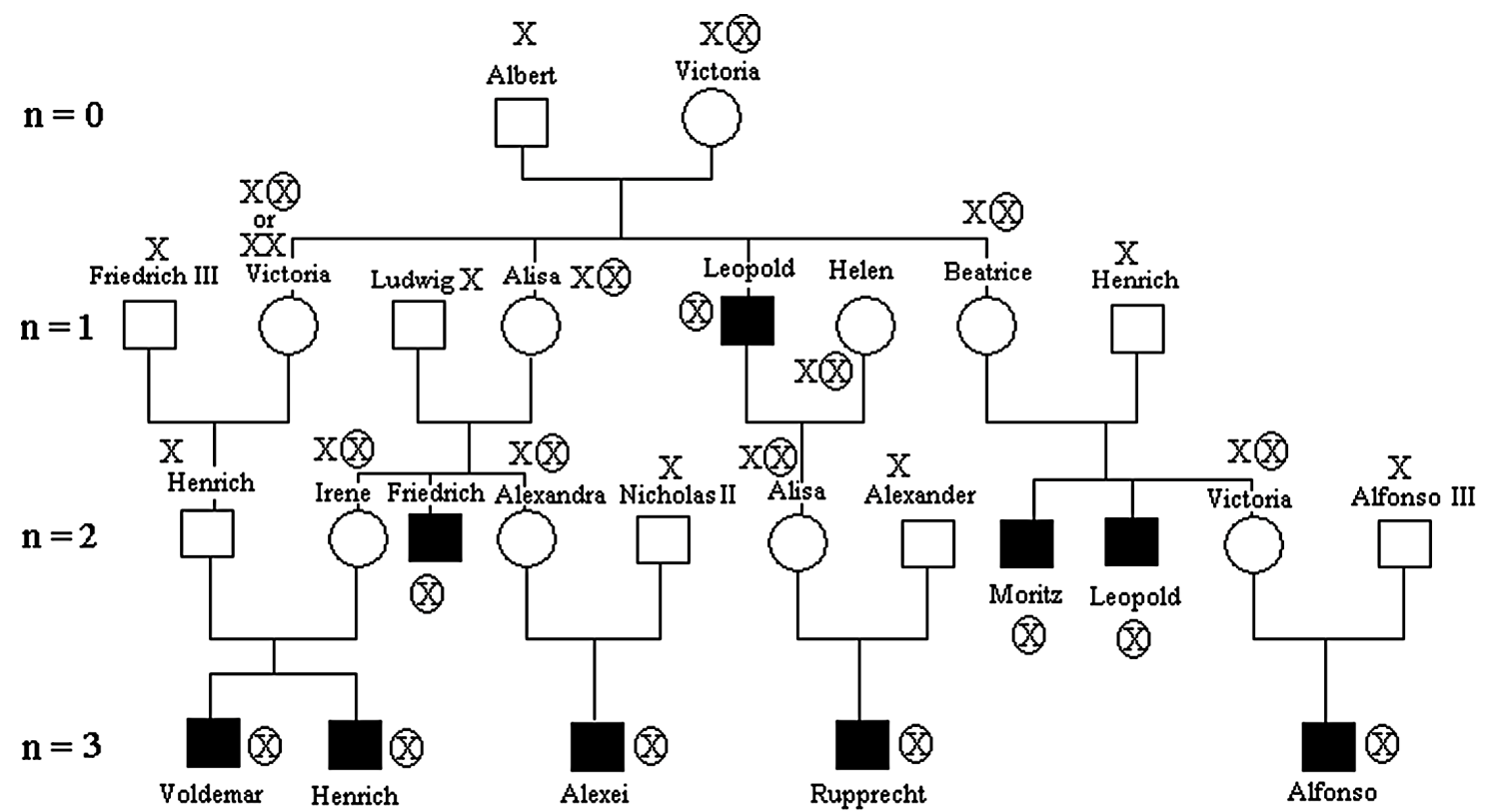

Figure 4. Family tree of the European royal houses in which men frequently were sick of hemophilia.

preservation of Hardy-Weinberg equilibrium on some level i.e. ratio of genotypes frequencies (1).

The balance at hemophilia mutagenesis and selection allows find connection between the equivalent constant mutagen factor $b$ and standard parameter of selection $s$ [2].

Let's consider selection against homozygotes $a a$. In [5], there is concrete example of such selection - the phenomenon of industrial melanism, i.e. change of painting of butterfly Biston betularia in industrial regions of England is resulted at the end of XIX century.

Genotypes before selection, for example, in generation $n-2$ are distributed according to (1).

We accept fitness of genotypes [2]:

$$
1: 1:(1-s)
$$

where $s$ there is reduction of homozygotes fraction of recessive alleles as a result of selection (selection parameter).

Genotypes after selection we shall write down for the following generation $(n-1)$ :

$$
\begin{aligned}
& (A A) p_{f(n-1)} p_{m(n-1)}:(A a)\left(p_{m(n-1)} q_{f(n-1)}\right. \\
& \left.+p_{f(n-1)} q_{m(n-1)}\right):(a a) q_{m(n-1)} q_{f(n-1)}(1-s)
\end{aligned} .
$$

Taking into account, that

$$
\begin{aligned}
& p_{f(n-1)} p_{m(n-1)}+\left(p_{m(n-1)} q_{f(n-1)}+p_{f(n-1)} q_{m(n-1)}\right) \\
& +q_{m(n-1)} q_{f(n-1)}=\left(p_{f(n-1)}+q_{f(n-1)}\right)\left(p_{m(n-1)}+q_{m(n-1)}\right)=1
\end{aligned},
$$

we find the sum of frequencies of genotypes:

$1-q_{m(n-1)} q_{f(n-1)} s$.
Further, using a standard rule of a finding of alleles frequency in the following generation (half of heterozygotes frequency plus of homozygotes frequency) and the formula (14) we calculate the frequency of recessive alleles $a$ at women in generation $n$ it is similar [2] where such calculation is made for autosomal genome:

$$
\begin{aligned}
& q_{f n} \\
&= \frac{1}{2}\left(p_{m(n-1)} q_{f(n-1)}+p_{f(n-1)} q_{m(n-1)}\right)+q_{m(n-1)} q_{f(n-1)}(1-s) \\
& 1-q_{m(n-1)} q_{f(n-1)} s
\end{aligned} .
$$

Let's transform the formula (15), using

$$
\begin{gathered}
p_{m(n-1)}=1-q_{m(n-1)} \text { and } p_{f(n-1)}=1-q_{f(n-1)}: \\
q_{f n}=\frac{\frac{1}{2}\left(q_{f(n-1)}+q_{m(n-1)}\right)-q_{m(n-1)} q_{f(n-1)} s}{1-q_{m(n-1)} q_{f(n-1)} s} .
\end{gathered}
$$

At selection all changes in a population occur due to size $s$ therefore we believe that alleles $a$ frequency at the man is equal to alleles $a$ frequency at the woman of the previous generation $q_{m(n-1)}=q_{f(n-2)}$. Hence:

$$
q_{f n}=\frac{\frac{1}{2}\left(q_{f(n-1)}+q_{f(n-2)}\right)-q_{f(n-2)} q_{f(n-1)} s}{1-q_{f(n-2)} q_{f(n-1)} s} .
$$

As it is underlined in [2], Eq.17 has no general solution even for autosomal genome. Especially it is not present for genome linked with the $X$-chromosome. It is essentially complicates the mathematical analysis of the Eq.17. First of all does not allow is proved that the size $s$ re- mains constant from generation to generation. 
Mutagenesis and selection act in one direction. If arisen for the account mutagenesis destructive alleles will be eliminated from the family tree with the same speed due to selection the balance to be kept. A quantity of the destructive genes is kept in a family tree but this quantity will not increase.

We assume in process of mutagenesis in generation $n$ at women the quantity of the destructive alleles $\mathrm{O}$ has increased in comparison with generation $(n-1)$. We assume generation $n$ where the constancy $\Delta q_{f(n-1)}$ was already established, Figure 3. Frequency of destructive alleles has increased on $\Delta q_{f(n-1)}=q_{f n}-q_{f(n-1)}$. These alleles due to selection eliminate from a family tree. The balance of mutagenesis and selection will arise in case of equality of frequency $q_{f n}$ in mutagenesis and selection since frequency $q_{f(n-1)}$ up to a mutation is supposed in both cases identical.

Taking into account balance mutagenesis and selection, we equate (5) and (17). After simple transformations, we find:

$$
\begin{aligned}
b & =1-q_{f(n-1)}\left(2-q_{f(n-1)}-b q_{f(n-2)}\right) s \\
& =1-2 q_{f(n-1)} p_{f n} s
\end{aligned}
$$

In a case with hemophilia at the mutagen factor $b=0.8$ and identical genic frequencies at men and the women equal 0.6057 standard parameter of selection designed under the formula (18) $s=0.27$, and fitness of a genotype $1-s=0.73$. The estimation of fitness is enough challenge. One of possible ways to calculate fitness to find it is as the ratio of an average of survived children of the falling one parent which is sick of hemophilia to an average of survived children on one healthy parent. For example, in [6] the opportunity of calculation is offered (though and with reserves) to fitness at hemophilia $1-s=1.75 / 2.5=0.7$.

Thus, the opportunity of the accuracy solution of the equation describing change of frequencies in generations with the help of the constant mutagen factor $b$ as against use of the $s$-dependent Eq.17 apparently specifies preferable of value $b$.

At the analysis of the accuracy solution of process of mutagenesis, for example, in a case with hemophilia, there is an opportunity of more reliable calculation of constant mutagen factor size if there are authentic data on a full family tree with destruct of corresponding allele.

\section{ACTION OF THE CONSTANT MUTAGEN FACTOR ON A POPULATION}

In the previous section, action of the constant mutagen factor on a genome of a family tree has been considered. Besides, it has been marked that spontaneous mutagene- sis at balance of mutations and selection (in particular at hemophilia), it is possible to present as action of the equivalent constant mutagen factor.

In the present section, we will analyze action of the constant mutagen factor (mobile communication, radiation of office equipment, etc.) on a human population.

The Hardy-Weinberg law in the kind considered above there is concerns to a separate family tree. Implicitly this law includes time since alternation of generations occurs through certain period. Average time of a life of one generation is $T=25-30$ years. Thus Hardy-Weinberg law has definitely discrete character on time. The population will consist of family trees crossed among themselves and lives in continuous time. Alternation of generations into family trees set results to that generations vary actually according to a continuous time scale.

Therefore we shall pass to a continuous scale of generations $n$. Under size $n$ in this case we mean time of a life of the population normalized on average on a population time of a life of one generation, i.e. actually continuous dimensionless time.

The analysis we shall make on the basis of HardyWeinberg law written down as [4]:

$$
\frac{\mathrm{d}^{2} q_{f(n-1)}}{\mathrm{d} n^{2}}+\ln 2 \frac{\mathrm{d} q_{f(n-1)}}{\mathrm{d} n}=\alpha,
$$

where the value $\alpha$ there is characterizes some constant mutagen factor.

The Eq.19 can be integrated once:

$$
\frac{\mathrm{d} q_{f(n-1)}}{\mathrm{d} n}+\ln 2 q_{f(n-1)}=\alpha n+C_{1},
$$

where $C_{1}$ there is a constant of integration.

The Eq.20 is integrated in quadratures. The general solution looks like:

$$
q_{f(n-1)}=\frac{\alpha n}{\ln 2}-\frac{\alpha}{(\ln 2)^{2}}+\frac{C_{1}}{\ln 2}+C_{2} \mathrm{e}^{-n \ln 2},
$$

where $C_{2}$ there is a constant of integration.

Change of alleles $a$ frequency for one generation is equal:

$$
\begin{aligned}
\Delta q_{f(n-1)} & =q_{f n}-q_{f(n-1)} \\
& =\frac{\alpha(n+1)}{\ln 2}-\frac{\alpha}{(\ln 2)^{2}}+\frac{C_{1}}{\ln 2}+C_{2} \mathrm{e}^{-(n+1) \ln 2} \\
& -\left(\frac{\alpha n}{\ln 2}-\frac{\alpha}{(\ln 2)^{2}}+\frac{C_{1}}{\ln 2}+C_{2} \mathrm{e}^{-n \ln 2}\right) \\
& =\frac{\alpha}{\ln 2}-\frac{1}{2} C_{2} \mathrm{e}^{-n \ln 2}
\end{aligned}
$$

At increase in number of generations (or time) $n \rightarrow \infty$ the change of alleles frequency $\Delta q_{f \infty}=\frac{\alpha}{\ln 2}$. 
Let's address now to the analysis of decrease of alleles blood $\mathrm{O}$ frequency at there is hemophilia. As it was specified earlier a spontaneous mutation at hemophilia it is possible to present as action of some constant equivalent mutagen factor, in this case $\alpha$.

Frequency of mutations at hemophilia in different countries (on a population) changes from $4.4 \times 10^{-5}$ (Switzerland), up to $6.4 \times 10^{-5}$ (Denmark), i.e. a gene of a hemophilia have from 44 up to 64 women on one million [2]. Frequency of mutations it is ratio of number of cases of anomaly display to the double number of the examined individuals the corrected sizes of frequencies of mutations therefore are used.

Let at $n \rightarrow \infty$ the size is $\Delta q_{f \infty} \approx-6 \times 10^{-5}$. I.e. 60 girls which birth from one million have a gene of a hemophilia. In this case the equivalent constant mutagen factor $\alpha=\Delta q_{f \infty} \ln 2 \approx-4.16 \times 10^{-5}$.

The basic uncertain size in dynamics of change of alleles frequencies (22) is the constant $C_{2}$. According to (22), we shall find the law of decrease in genic frequency:

$$
q_{f n}=q_{f(n-1)}+\frac{\alpha}{\ln 2}-\frac{1}{2} C_{2} \mathrm{e}^{-n \ln 2} .
$$

We use the initial condition: at $n=1$ according to (3) $q_{f n}=q_{f 1}=\frac{q_{m 0}+q_{f 0}}{2}$.

Hence:

$$
q_{f 1}=q_{f 0}+\frac{\alpha}{\ln 2}-\frac{1}{4} C_{2}=\frac{q_{m 0}+q_{f 0}}{2} .
$$

From (24) we find constant $C_{2}$ :

$$
C_{2}=2\left(q_{f 0}-q_{m 0}\right)+\frac{4 \alpha}{\ln 2} .
$$

Substituting (25) in (22), we shall find:

$$
\Delta q_{f(n-1)}=\frac{\alpha}{\ln 2}-\left(\left(q_{f 0}-q_{m 0}\right)+\frac{2 \alpha}{\ln 2}\right) \mathrm{e}^{-n \ln 2} .
$$

Believing as well as in case of a family tree $q_{f 0}=q_{m 0}$, we shall receive:

$$
\Delta q_{f(n-1)}=\frac{\alpha}{\ln 2}\left(1-2 \mathrm{e}^{-n \ln 2}\right)=\frac{\alpha}{\ln 2}\left(1-2^{-(n-1)}\right) .
$$

At $n=0$ we find initial change of alleles $\mathrm{O}$ frequency which is equal $\Delta q_{f(-1)}=-\frac{\alpha}{\ln 2}$. Taking into account $\Delta q_{f(-1)}=-\frac{\alpha}{\ln 2}$ we find $\Delta q_{f(-1)} \approx 6 \times 10^{-5}$. We shall note that the value $\Delta q_{f(-1)}$ has rated character. Change of genic frequency is real at mutagenesis begins from time coordinate $n=1$ at which according to (27) $\Delta q_{f(n-1)}=\Delta q_{f(0)}=0$.

On Figure 5, the dependence of change of genic alleles $\mathrm{O}$ frequency $\Delta q_{f(n-1)}$ at women from time of the population life plotted under the formula (27) is shown.

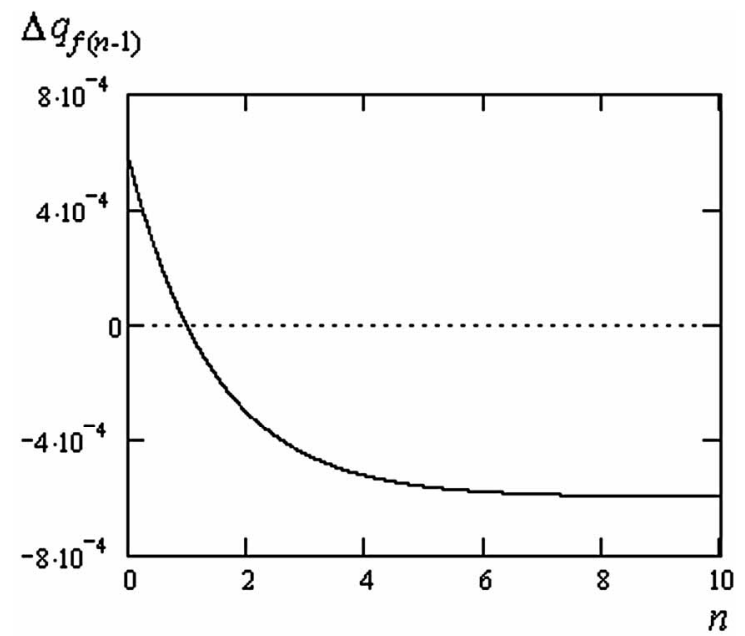

Figure 5. Dependence of change of genic frequency $\Delta q_{f(n-1)}$ of blood allele $\mathrm{O}$ at women from time of a population life.

Comparison about Figure 3 for change of genic alleles $\mathrm{O}$ frequency (under condition of the equivalent constant mutagen factor action) plotted for a separate family tree shows that as whole dependences are similar. Smoothness of a curve on Figure 5 is defined by a continuity of a scale alternation of generations or dimensionless time for a population as against discrete time of a separate family tree.

The analysis of Figure 5 allows to assume that under action of the equivalent constant mutagen factor $\alpha$ acting on a population, there is average on species of a population the mutation resulting in average on a population to continuous reduction of frequency of alleles $\mathrm{O}$ healthy at women and during too time to increase at them of frequency of alleles $\mathrm{O}$ destructive i.e. to a hemophilia. Action of selection, for example, decrease in life period of men with hemophilia on $1 / 3$ and decrease in their birth rate are compensates reduction of destructive alleles frequency [2]. In this the dynamics of development of hemophilia in a population as a whole does not differ from those in a family tree.

\section{ACTION OF SELECTION ON A POPULATION}

Let's consider action of selection on a population. Using (17) the change of alleles $a$ frequency in a family tree it is possible to calculate under the formula:

$$
\begin{aligned}
& \Delta q_{f(n-1)}=q_{f n}-q_{f(n-1)} \\
& =\frac{\frac{1}{2}\left(q_{f(n-1)}+q_{f(n-2)}\right)-q_{f(n-2)} q_{f(n-1)} s}{1-q_{f(n-2)} q_{f(n-1)} s}-q_{f(n-1)} \\
& =\frac{\frac{1}{2}\left(-q_{f(n-1)}+q_{f(n-2)}\right)-q_{f(n-2)} q_{f(n-1)} s+q_{f(n-2)} q_{f(n-1)}^{2} s}{1-q_{f(n-2)} q_{f(n-1)} s}
\end{aligned}
$$


At transition to a continuous time scale we believe difference in genic frequencies of two generations following one after another infinitesimal, i.e. $q_{f(n-2)} \approx q_{f(n-1)}$. Hence, the formula (28) will be transformed to a kind similar autosomal genome [2]:

$$
\Delta q_{f(n-1)}=-\frac{q_{f(n-1)}^{2} s\left(1-q_{f(n-1)}\right)}{1-q_{f(n-1)}^{2} s} .
$$

At small frequencies of alleles $a$ can be approximated change of frequency (actually the differential) by expression [2]:

$$
\Delta q_{f(n-1)}=-q_{f(n-1)}^{2} s\left(1-q_{f(n-1)}\right)=-q_{f(n-1)}^{2} s p_{f(n-1)},
$$

where $p_{f(n-1)}=1-q_{f(n-1)}$ there is frequency of dominant alleles $A$ at women. For blood system ABO $p_{f(n-1)}$ there is total frequency of blood dominant alleles.

If process of selection is stationary it is possible to use the frequencies of alleles at $n \rightarrow \infty$ :

$$
\Delta q_{f \infty}=-q_{f \infty}^{2} p_{f \infty} s .
$$

Actually, this formula accordance with Figure 5 it is valid already at $n>8$.

Thus, the parameter of selection $s$ in a population can be calculated under the formula:

$$
s=-\frac{\Delta q_{f \infty}}{q_{f \infty}^{2} p_{f \infty}} .
$$

Let's note, that parameter of selection $s$ it is size positive since $\Delta q_{f_{\infty}} \leq 0$. The alleles $a$ frequency is reduced.

Using the formula (27) at $n \rightarrow \infty$ we find

$\Delta q_{f \infty}=\frac{\alpha}{\ln 2}$. Substituting this expression in (32) we shall find connection between parameter of selection $s$ and the characteristic of the constant equivalent mutagen factor $\alpha$ in a population:

$$
\alpha=-s q_{f \infty}^{2} p_{f \infty} \ln 2 .
$$

Apparently from (33), there is a linear connection be- tween parameters $s$ and $\alpha$ that confirms of Danforth and Haldane ideas about an opportunity of mutagenesis and selection balance at presence of hemophilia.

\section{CONCLUSION}

Hereditary disease hemophilia can be analyzed as one example of separate family tree, and all populations as whole. For a quantitative estimation on the basis of Hardy-Weinberg law, allele blood $\mathrm{O}$ frequency changes can be used as the equivalent constant mutagen factor and it is also an idea of Danforth and Haldane about balance of mutagenesis and selection at presence of hemophilia. As the whole the analysis of a family tree on example of the European royal houses and populations leads to the same basic results, change of allele frequencies after some lives of a population (or generations in a family tree) becomes constant. Selection compensates the given change of allele frequencies.

\section{REFERENCES}

[1] von E. Libbert, H. (1982) Kompendium der allgemeinen biologie. VEB Gustav Ficher Verlag, Jena, 438 p.

[2] Vogel, F. and Motulsky, A. (1990) Human genetics. 1, 310 p, 2, 380 p. Springer-Verlag, Berlin.

[3] Volobuev, A.N. and Petrov, E.S. (2011) Modelling of the populating development of the genome in the radiation of the environment. Natural Science, 3, 1029-1033. doi:10.4236/ns.2011.312128

[4] Volobuev, A.N., Romanchuk, P.I. and Malishev, V.K. (2013) About one form of writing of the Hardy-Weinberg law. Natural Science, 5, 724-728. doi:10.4236/ns.2013.56089

[5] Ayala, F. and Kiger Jr., J. (1984) Modern genetics. V. 3. The Benjamin/Cummings Publishing Company, Inc. California, $335 \mathrm{p}$.

[6] Li, C.C. (1976) First course in population genetics. The Boxwood Press Pacific Grove, California, 556 p. 